\title{
HAMARTOMA CÍSTICO RETRO-RETAL: RELATO DE 2 CASOS E REVISÃO DA LITERATURA
}

\author{
GABRIELLA OLIVEIRA FERNANDES ${ }^{1}$, PATRÍCIA ALVES MANGUEIRA ${ }^{1}$, CARLÚCIO CRISTINO PRIMO $^{1}$, \\ MARCO AURÉLIO VIANA FRANÇA ${ }^{1}$, JOSÉ HERMES GOMES COSTA ${ }^{1}$
}

${ }^{1}$ Serviço de Coloproctologia do Hospital Geral de Goiânia (H.G.G.), Goiânia - Goiás, Brasil

FERNANDES GO, MANGUEIRA PA, PRIMO CC, FRANÇA MAV, COSTA JHG. Hamartoma Cístico Retro-retal: Relato de 2 casos e revisão da literatura. Rev bras Coloproct, 2006;26(2):178-186.

RESUMO: Tumores retro-retais são aqueles localizados no espaço retro-retal, de diferentes origens embriológicas e que podem exercer compressão extrínsica no reto e canal anal. São lesões raras, geralmente assintomáticas e mais comuns em mulheres. $O$ hamartoma cístico (tailgut cyst) é um tipo de tumor retro-retal congênito, formado a partir de remanescentes embrionários do intestino posterior. Este trabalho traz uma revisão sobre os tumores retro-retais, dando ênfase às lesões císticas e relata 02 casos de pacientes com hamartoma cístico retro-retal, tratados no Hospital Geral de Goiânia,com lesões evidentes ao toque retal e exames de imagem mostrando lesões císticas no espaço pré-sacral. Utilizou-se a incisão de Parks na abordagem das lesões, uma delas associada 'a via abdominal. A histopatologia foi compatível com hamartoma cístico. As lesões císticas do espaço retro-retal possuem bom prognóstico e a abordagem cirúrgica dependerá das características da lesão, principalmente sua altura em relação à margem anal e relação com estruturas adjacentes no espaço pré-sacral.

Descritores: Hamartoma cístico; tailgut cyst; tumor retro-retal

\section{INTRODUÇÃO}

Os hamartomas císticos (HC) são tumores provenientes de vestígios embrionários caudais (intestino posterior) ${ }^{6,10,12,13}$, em geral multiloculados, de parede delgada, internamente de coloração amarelada, preenchidos por secreção espessa, mucóide e clara, até um fluido opaco amarelo-esverdeado 9 . São revestidos por uma variedade de epitélios, mas a presença de epitélio colunar ou de transição é necessária para distinguí-los dos cistos dermóide e epidermóide ${ }^{1,7,8,9,12,13}$. Apresentam à microscopia células secretoras de mucina ou células colunares ciliadas, células escamosas e células transicionais, algumas vezes em combinação. ${ }^{2,6,9}$. Podem acometer crianças e são mais comuns em mulheres de meia-idade, algumas vezes diagnosticados em ultrassonografias(USG) ginecológicas, exame pré-natal ou exames médicos de rotina, tornando-se um achado ocasional $1,6,9,13,19,21$. O objetivo deste trabalho é apresentar 02 casos de HC retro-retais e revisar a literatura sobre o assunto.

Trabalho realizado no Serviço de Coloproctologia do Hospital Geral de Goiânia (H.G.G.) , Goiânia - Goiás,Brasil

$\overline{\text { Recebido em } 04 / 04 / 2006}$

Aceito para publicação em 17/05/2006 


\section{DESCRIÇÃO DOS CASOS}

\section{Caso 01}

L.A.S., 23 anos, sexo feminino, com queixa de desconforto retal ao evacuar, sensação de evacuação incompleta e dor na região glútea esquerda, irradiada para a face posterior da coxa homolateral. Durante consulta ginecológica, evidenciou-se a partir de USG transvaginal, lesão nodular hipoecogênica com sombra acústica posterior, na transição reto-vaginal, de aproximadamente $7,4 \mathrm{~cm}$ no maior diâmetro, sugestiva de endometrioma (Figura-1). Encaminhada ao Serviço de Coloproctologia do Hospital Geral de Goiânia (H.G.G.), o exame proctológico mostrou lesão extrínsica comprimindo a parede retal pósterolateralmente à esquerda, a mais ou menos $8 \mathrm{~cm}$ da margem anal, de consistência fibro-elástica e depressível, com mucosa retal íntegra. A Colonoscopia mostrou abaulamento extrínsico da parede posterior do reto, a $10 \mathrm{~cm}$ da margem anal (Figura-2). Foi realizada Ressonância Magnética (RM) de abdome inferior, que identificou lesão cística volumosa no espaço retro-retal, de margem cefálica ao nível de S3S4 e caudal abaixo do cóccix, comprimindo reto, vagina e útero anteriormente, medindo $10 \times 9 \times 6 \mathrm{~cm}$ (Figura-3). Ausência de alterações ósseas. A paciente foi submetida à intervenção cirúrgica, com acesso abdominal e perianal interesfincteriano através de incisão de Parks (Figura-4), com exérese de lesão

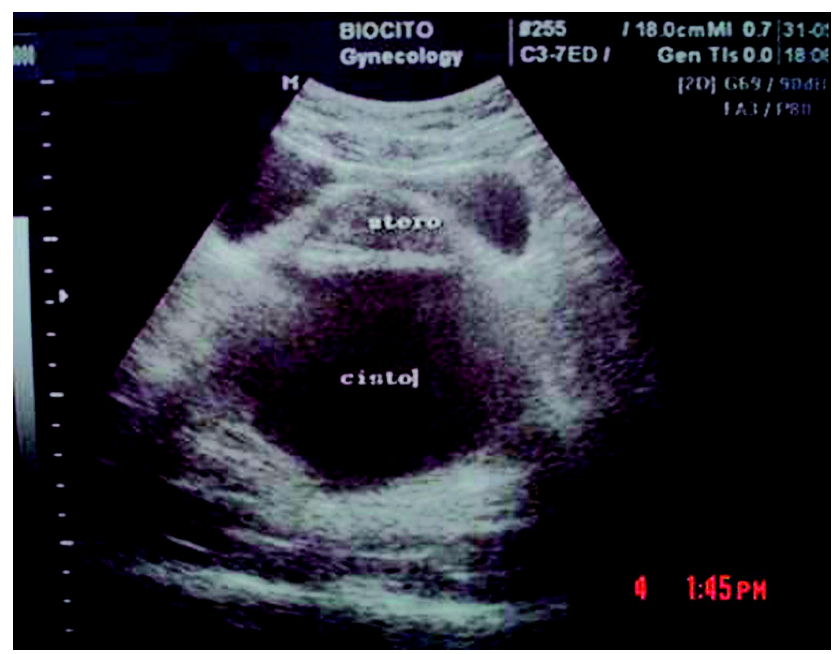

Figura 1 - USG transvaginal, com lesão cística.

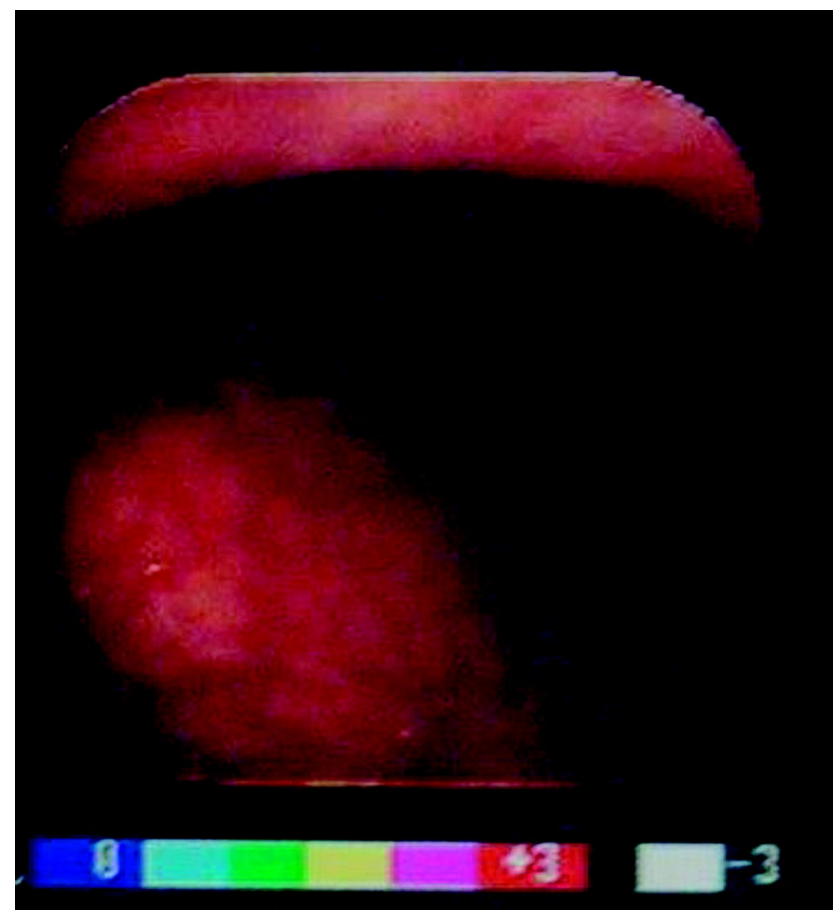

Figura 2 - Colonoscopia mostrando lesão extrínsica posterior à parede retal.

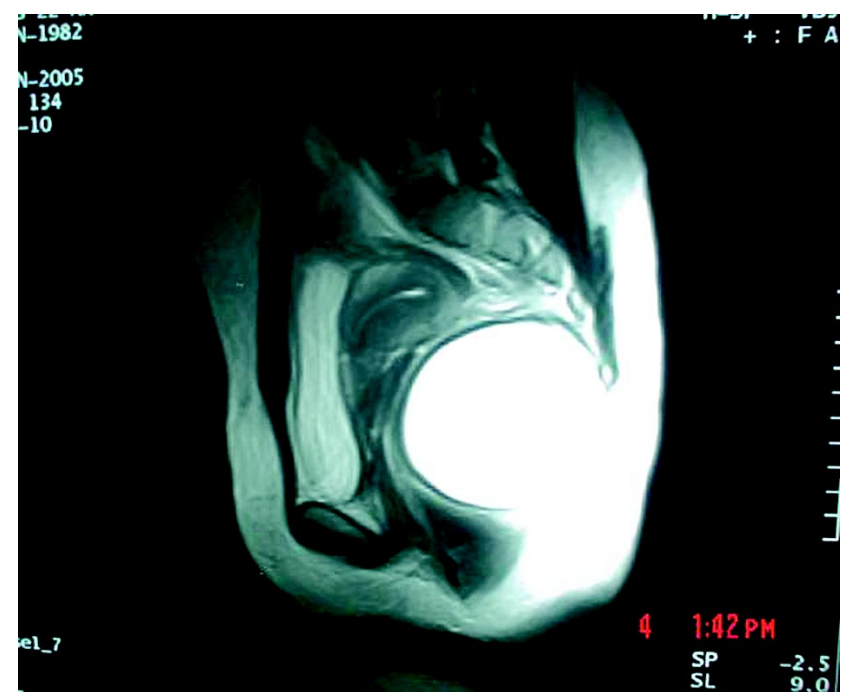

Figura 3-RM com lesão cística retro-retal volumosa.

cística retro-retal (Figuras-5 e 6). Pós-operatório sem intercorrências. Histopatológico mostrou estrutura cística revestida parcialmente por epitélio escamoso, com intensa infiltração de mononucleres e histiócitos (Figura-7), compatível com HC retro-retal. A paciente permanece em acompanhamento, mantendo-se há 10 meses sem sinais de recidiva. 


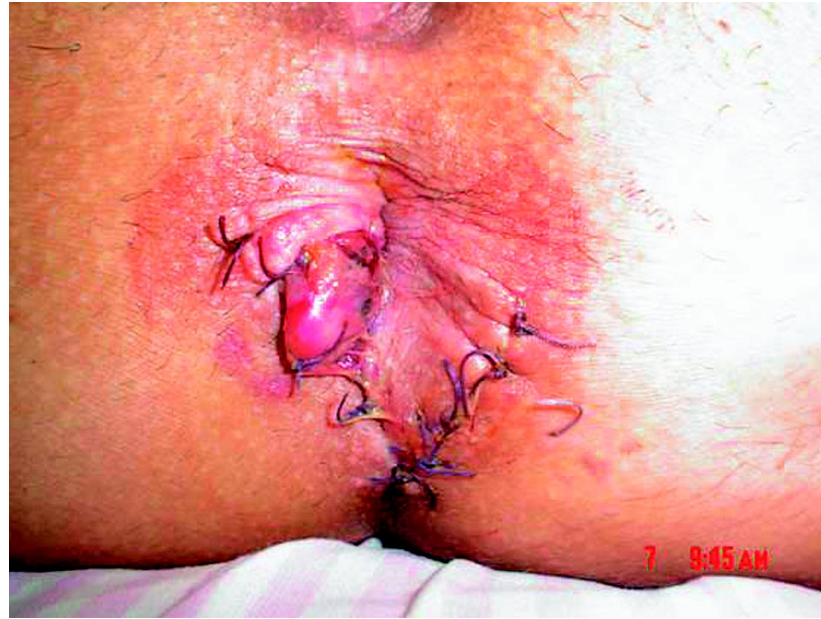

Figura 4 - Abordagem perianal com incisão de Parks.

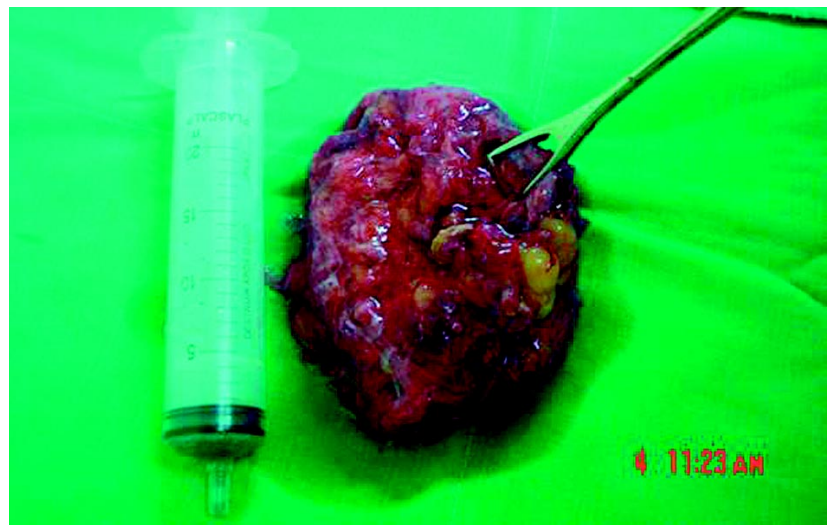

Figura 5 - Lesão cística.

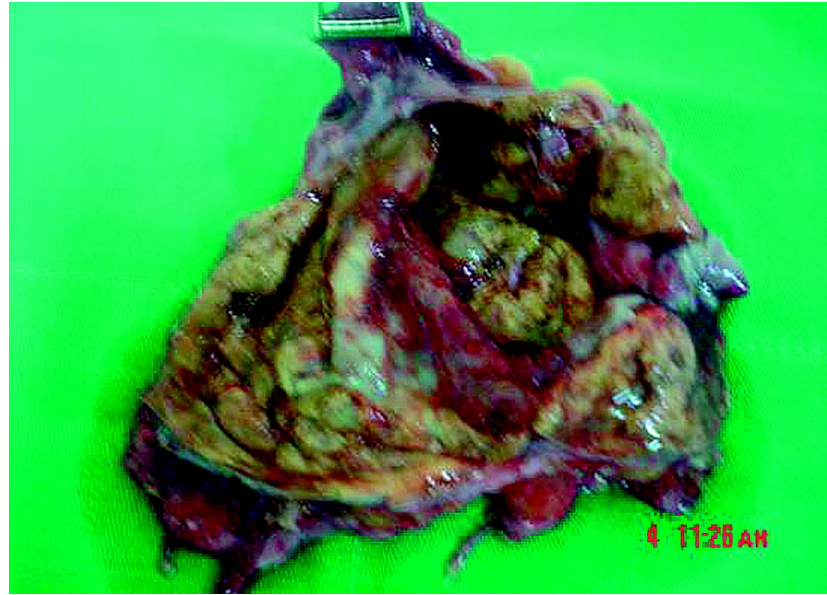

Figura 6 - Lesão cística seccionada, com parede interna amarelada, típica de $\mathrm{HC}$.

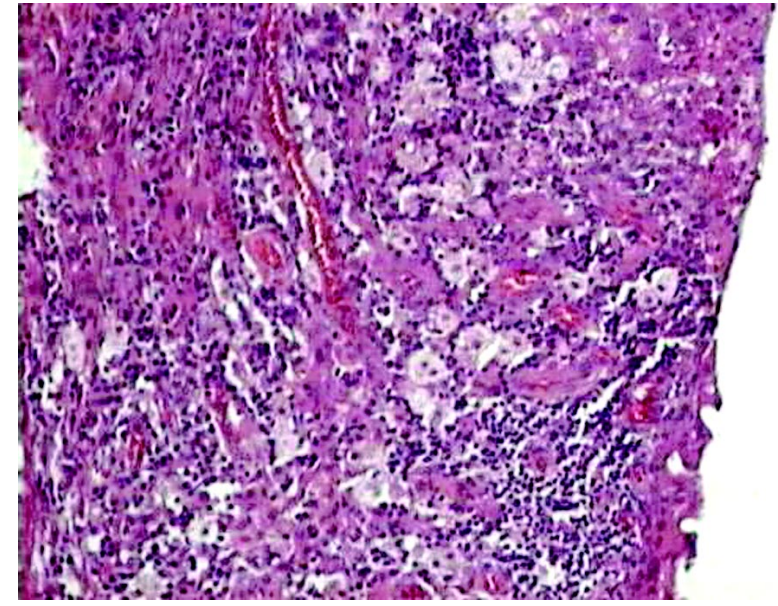

Figura 7 - Análise histopatológica da lesão.

\section{Caso 02}

M.U.D.B., 33 anos, sexo feminino, procurou o ambulatório do Serviço de Coloproctologia do H.G.G., queixando-se de dor em nádega direita, com abaulamento local progressivo há + - 3 meses, além de dor às evacuações. Realizou USG em nádegas, que mostrou imagem cística bilobulada, estendendo-se da região coccígea até a margem anal direita, de aproximadamente $8 \mathrm{~cm}$ no maior diâmetro, sugestiva de cisto pilonidal (Figura-8). A inspeção e palpação perineal mostraram tumoração na margem anal direita, prolongando-se em direção à nádega homolateral até $+-4 \mathrm{~cm}$ do intróito anal. Ao toque retal, a lesão seguia

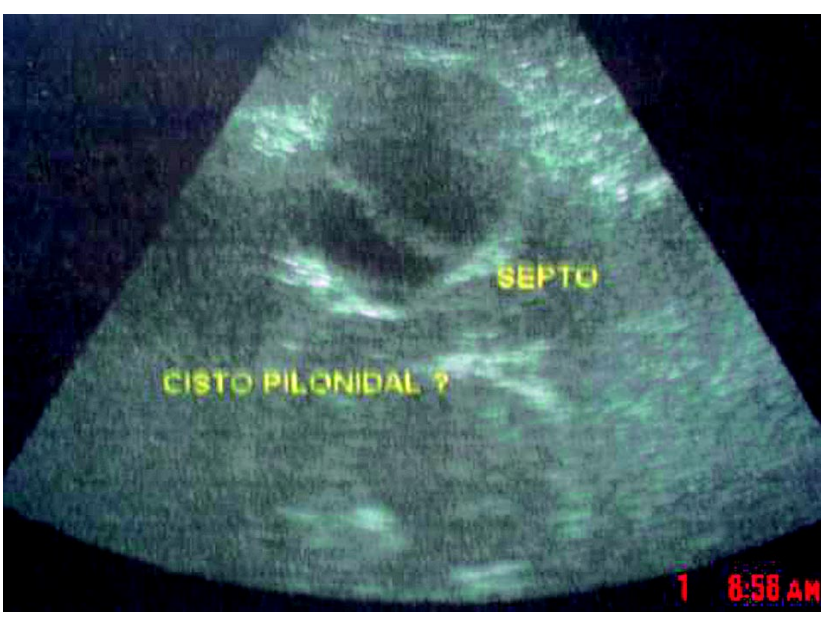

Figura 8 - USG sugerindo cisto pilonidal. 
cranial e posteriormente no canal anal, até $+-2 \mathrm{~cm}$ da linha pectínea, com mucosa normal, registrada pela Colonoscopia (Figura-9). Tomografia Computadorizada (TC) de abdome inferior mostrou lesão cística bilobulada no espaço retro-retal, extrínsica ao reto, comprimindo o canal anal e rechaçando a nádega direita (Figura-10). A paciente foi submetida à cirurgia de abordagem perianal interesfincteriana (incisão de Parks)(Figura-11), com ressecção da lesão (Figuras12 e 13). Evoluiu sem intercorrências em pósoperatório. Histopatológico evidenciou lesão cística revestida internamente por epitélio escamoso e mucosecretor, com erosões, compatível com HC (Figura 14). Seguimento de 8 meses, sem evidências de recidiva.

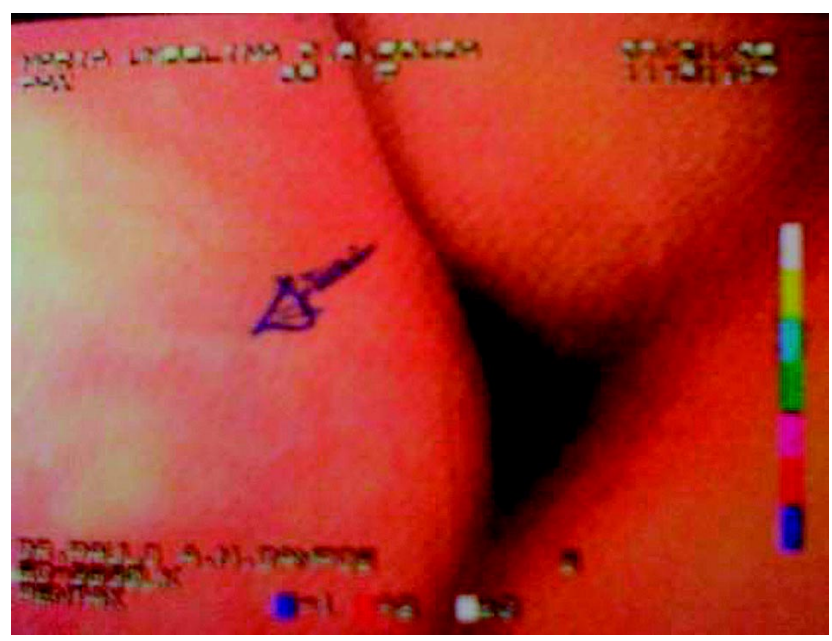

Figura 9 - Colonoscopia com abaulamento extrínsico da parede retal.

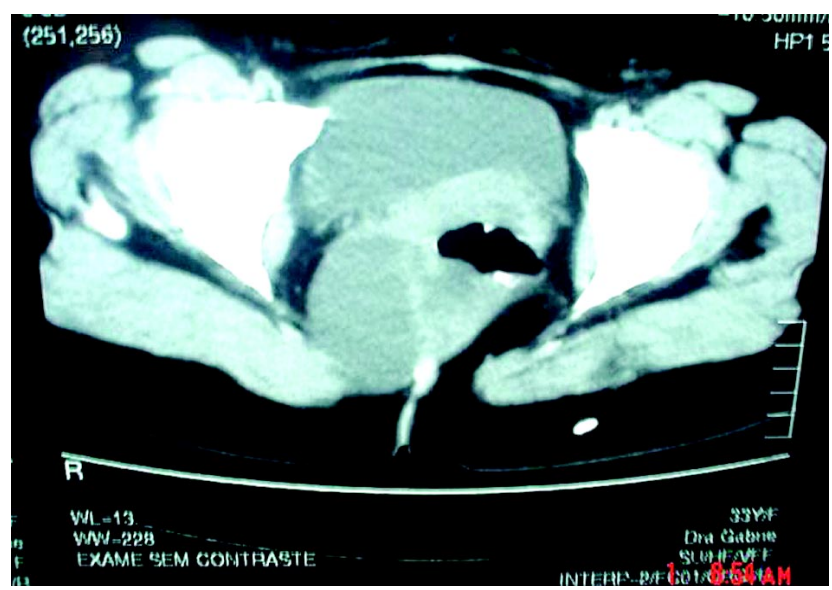

Figura 10 -Imagem cística retro-retal bilobulada na TC de abdome.

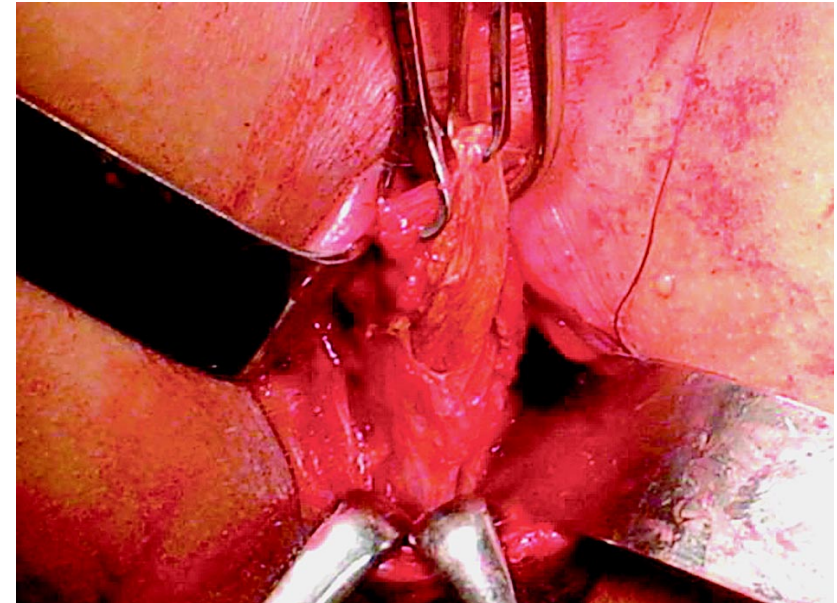

Figura 11 - Abordagem perianal à Parks

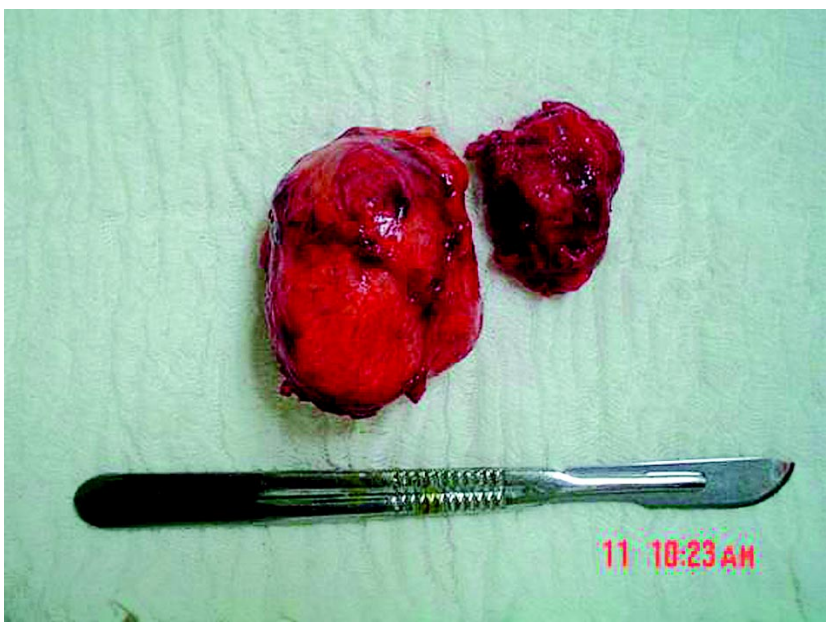

Figura 12 - Lesão cística bilobulada ressecada.

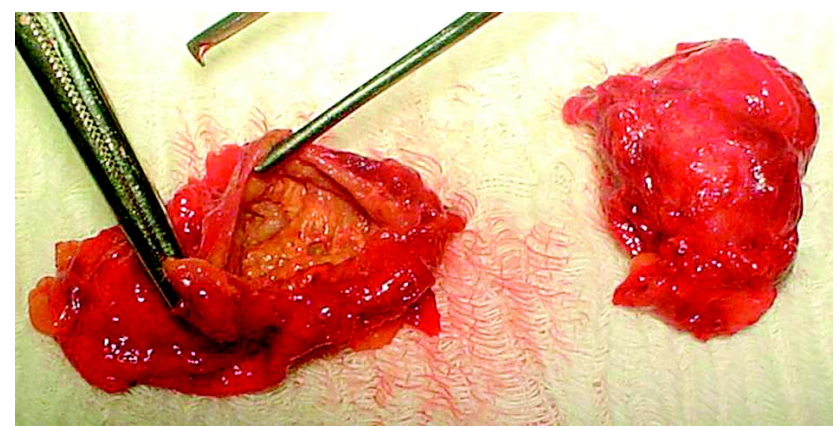

Figura 13 - Lesão seccionada. 


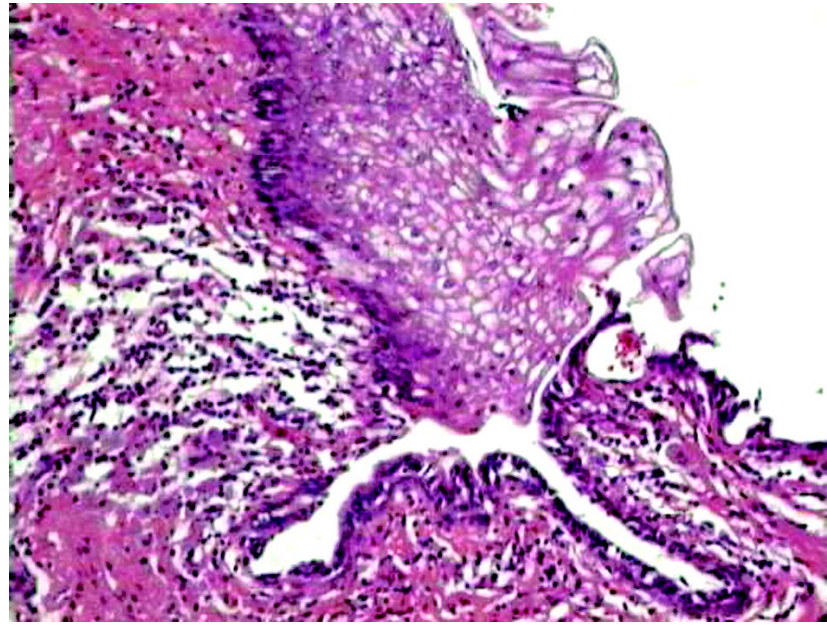

Figura 14 - Exame histopatológico compatível com H.C.

\section{DISCUSSÃO}

Os tumores retro-retais são lesões raras, constituindo um grupo heterogêneo do ponto de vista histo-embriológico, localizados no espaço retro-retal ou pré-sacral?. Os limites desse espaço são : anterior reto e sua fáscia; posterior - fáscia reto-sacral de Waldeyer; laterais - vasos hipogástricos, ureteres e ligamentos laterais do reto; superior - reflexão peritoneal ao nível de S2-S3; e inferior - os músculos elevadores ${ }^{3,9,21,23}$.

O primeiro relato de tumor do espaço retroretal foi feito por Emmerich em 1847 e se tratava de um teratoma ${ }^{3}$. Em função da presença de estruturas embriológicas neste espaço, como o intestino posterior, o neuroectoderma e precursores dos ossos pélvicos, torna-se factível a formação de tumores benignos e malignos locais, de natureza notocordal, cloacal, neurogênica, dentre outras ${ }^{1,3}$. A persistência de vestígios embriológicos da chamada "cauda intestinal" (tailgut) dará origem aos $\mathrm{HC}$, também conhecidos como cistos do intestino posterior, hamartoma cístico mioepitelial, cisto embrionário secretor de mucina, vestígio do intestino posterior, cisto retal ou tailgut cyst $\mathrm{t}^{13,14,15,21,23}$.

A classificação geral das lesões retro-retais compreende: lesões congênitas ou embriológicas, neurológicas, ósseas e miscelânias (Tabela-1) ${ }^{11,20,21}$. Os cistos embriológicos do espaço retro-retal se classificam em : cistos dermóides, cistos epidermóides, cistos neuroentéricos e cistos entéricos (Tabela-2). Dentre os últimos estão os $\mathrm{HC}^{2}$.
Tabela 1 - Classificação dos tumores retro-retais.

\section{ETIOLOGIA}

Congênita ou de desenvolvimento

Neurogênica

Óssea

Miscelâneas

Tabela 2 - Classificação das lesões císticas retro-retais

Cistos embrionários

- Cisto epidermóide

- Cisto dermóide

- Cistos neuroentéricos

- Cistos entéricos

- Hamartoma cístico

- Cisto de duplicação retal

Por serem lesões raras, sua verdadeira incidência e predominância histológica não estão claramente definidas, dificultadas ainda pela inclusão de lesões em estatísticas de tumores sacrais e neurológicos ${ }^{1,3,20,23}$. Sabe-se que os cistos embriológicos 
são as lesões congênitas mais comuns do espaço retroretal e, dentre os tumores malignos, os cordomas são o tipo histológico mais frequente ${ }^{1,11}$. Em crianças, o teratoma cístico é a lesão mais comum9

Em uma meta-análise combinada, descrita por Garcia-Aguilar ${ }^{21}$, em sete séries publicadas houve uma incidência de tumores retro-retais congênitos compressivos em $63 \%$, inflamatórios em $8 \%$, neurogênicos $10 \%$, ósseos $7 \%$ e miscelâneas $12 \%$. O risco para malignidade foi de $60 \%$ em lesões sólidas e $10 \%$ nas lesões císticas. Jao e col. relataram 120 pacientes com tumores retro-retais tratados na Mayo Clinic no período de $20 \operatorname{anos}^{24}$. Na experiência da Cleveland Clinic, foram descritos 24 casos de tumores sacrais e retro-retais, entre os anos de 1980 a $1992^{25}$. A partir desses e outros estudos, sugere-se que são diagnosticados de 1,4 a 6,3 tumores retro-retais por ano em grandes centros de referência ${ }^{26}$, estimando-se em 1 diagnóstico para cada 40 a 63 mil admissões ${ }^{1,3,11,21,24}$, podendo ser maior a incidência se considerarmos as estatísticas de serviços menores ${ }^{26}$.

Os HC são mais comuns em mulheres e em $50 \%$ dos casos as lesões são assintomáti$\cos ^{1,2,3,4,6,13,14,20,21,23}$. Acredita-se que sua predominância no sexo feminino se dê ao fato de mulheres se submeterem a exames de rotina, principalmente ginecológicos, em maior frequiência que os homens ${ }^{26}$. Pacientes no período gestacional podem iniciar a sintomatologia, pelo aumento do volume abdominal, comprimindo o canal do parto. O acompanhamento clínico é importante até o final da gestação, devendo o parto ser realizado preferencialmente via abdominal, evitando-se complicações fetais e maternas no período expulsivo vaginal ${ }^{4,19}$.

Quando atingem dimensões significativas, as lesões causam efeitos de massa local, com sintomas proctológicos do tipo constipação intestinal, desconforto às evacuações, dor retal e sensação de evacuação incompleta, além de dor lombar e sacral 1,3,5,9,12,20,21. Quadros inicialmente sugestivos de cisto pilonidal, cisto ovariano e tumor sacral, são descartados após a abordagem cirúrgica e análise histopatológica da lesão $0^{1,14,15,16,17,18}$. Cisto de duplicação retal (outro tipo de cisto entérico retro-retal) pode causar sangramento se apresentar ilhas de tecido ectópico (mucosa gástrica, pancreática ou urotelial) ${ }^{1,2}$. Lesões maiores podem fazer compressão no trato urinário inferior, causando disúria e freqüência urinária9 ${ }^{9}$ Alterações no osso sacro e calcificações podem estar presentes, mas são raras, constituindo síndromes com anomalias anorretais e meningoceles ${ }^{1,2,7,13}$. Em quase $100 \%$ dos casos o toque retal identifica compressão extrínsica posterior na parede do reto e/ou canal anal, com mucosa inalterada ${ }^{5,8,9,12,20,21,26}$.

A principal complicação das lesões císticas é a infecção(30-50\%), com formação de abscessos e fístulas, sendo que as últimas, quando originadas de cistos retro-retais, não apresentam abertura ao nível da linha pectínea ${ }^{1,2,21}$. A transformação maligna dos cistos entéricos é estimada em $7 \%$. Tailgut cyst pode acompanhar tumores reto-anais e do espaço retro-retal, como carcinóides, carcinoma escamoso e adenocarcinomas 9,10,11,1315,16,21.

No diagnóstico dos tumores retro-retais, a radiografia simples da região é de difícil análise, pela presença de gases intestinais e partes moles. Porém, calcificações locais e destruição dos ossos sacro e cóccix podem sugerir malignidade ${ }^{3}$. Nas lesões císticas com sintomas proctológicos, a retossigmoidoscopia, colonoscopia e enema opaco podem mostrar compressão extrínsica ${ }^{2,11,12}$. Trajetos fistulosos entre o cisto e a pele, ou a luz ano-retal são definidos pelo enema opaco e fistulografia ${ }^{1,2}$. USG transvaginal e pélvica evidenciam imagens císticas, uni ou multiloculadas, de diagnóstico impreciso

Exames como TC e RM têm permitido melhor avaliação dos tumores retro-retais, sua extensão, consistência, invasão de estruturas adjacentes, bem como comprometimento de linfonodos locais, podendo raramente não elucidar o diagnóstico ${ }^{3,5,16}$. Os HC determinam imagens císticas, bem delimitadas, posterior ao reto e/ou canal anal, com plano de clivagem entre eles, multilobulados na maioria dos $\operatorname{casos}^{12}$. A USG endoretal vem sendo usada na definição diagnóstica dessas lesões, com alta sensibilidade quando associada à história clínica e exame físico. Debris inflamatórios intralesionais descaracterizam o líquido homogêneo dos cistos, podendo sugerir lesões malignas ${ }^{12,21}$.

A realização de biópsias diagnósticas é controversa, devido ao risco de contaminação, meningite, sangramento da artéria sacral, recidivas locais e disseminação de lesões malignas, exceto nas lesões irressecáveis, na tentativa de decidir a terapia adjuvante a ser adotada ${ }^{1,3,19,21}$.

Dentre os diagnósticos diferenciais dos hamartomas císticos estão : cisto de duplicação retal, 
cisto dermóide, cisto epidermóide sacrococígeo, meningocele sacral anterior, cistos de glândulas anais, endometrioma, leiomiossarcoma retal necrótico, linfangioma cístico, abscesso piogênico, cisto neurogênico e cordoma sacral necrótico ${ }^{1,9,18}$. Em estudo retrospectivo por Glasgow et al, endometrioma foi o diagnóstico inicial mais comum de lesões retro-retais ${ }^{1}$, fato ocorrido no segundo caso aqui descrito.

O tratamento de eleição dos tumores retroretais é o cirúrgico, prevenindo-se complicações, como degeneração maligna, infecção e compressão nervosa ${ }^{1,5,6,7}$. A localização de cistos em um espaço anatômico de difícil acesso levou a várias abordagens cirúrgicas, sendo a via de acesso decidida conforme a altura, tamanho da lesão e comprometimento de estruturas adjacentes ${ }^{3,4,5}$. A experiência do cirurgião e o conhecimento do espaço a ser abordado devem ser considerados na escolha do tipo de abordagem ${ }^{3}$. Independente do acesso cirúrgico, a limpeza do cólon pré-operatória é indispensável, evitando-se contaminações desnecessárias.

O acesso posterior exclusivo (operação de Kraske) é reservado às lesões estendidas do sacro à ponta do cóccix. O último pode estar envolvido nos teratomas císticos, cistos dermóides e cistos epidermóides , com necessidade de ressecção óssea e possibilidade de sangramento. Uma variante do acesso posterior é o transesfincteriano tipo York-Mason, indicado quando há extensão lesional à parede retal, porém com resultados funcionais pobres ${ }^{3}$.

A abordagem abdominal, associada ou não ao acesso perineal, é preferida em lesões altas, com características malignas, de dimensões maiores, invadindo órgãos adjacentes ou lesões recidivadas, permitindo o controle vascular e confecção de estomas, quando necessário ${ }^{1,3,20}$. Dentre as vantagens da via abdominal estão a identificação dos ureteres, vasos sacrais e melhor dissecção tumoral da parede do reto ${ }^{1,3}$. Nos casos aqui descritos, a incisão de Parks foi escolhida pela facilidade técnica, menor sangramento operatório e localização baixa dos cistos, com resultados satisfatórios, sendo associada à via abdominal apenas no primeiro caso, devido ao prolongamento cranial da lesão.

Abordagens cirúrgicas agressivas abdominoperineais, com ressecção sacral e tumoral em bloco, ou exérese de lesões volumosas com inflamação e aderência local, podem causar efeitos indesejáveis de ordem urológica, como incontinência e impotência, sendo prudente a ressecção unilateral e preservação dos plexos nervosos contra-laterais ${ }^{1,3,13}$. Lesões congênitas associadas como menigoceles devem ser tratadas, tendo importância a presença do neurocirurgião no ato operatório ${ }^{13,}$

A sobrevida de pacientes com lesões benignas é estimada em $100 \%{ }^{5,26}$. A recidiva dos cistos embrionários é de aproximadamente $15 \%$, mais comum nos teratomas ${ }^{24}$. $\mathrm{O}$ índice de recidiva decresce nas ressecções em bloco incluindo o cóccix, que é sítio microscópico comum de células neoplásicas, especialmente nos teratomas $\mathrm{O}$ seguimento dos pacientes deve ser realizado com toque retal a cada 3 meses durante o primeiro ano, e em seguida anualmente, além dos exames de imagem para o diagnóstico de recidivas ${ }^{13}$.

\section{CONCLUSÕES}

Os HC retro-retais são lesões raras congênitas, mais comuns em mulheres, assintomáticas até que exerçam efeito de massa local, causando sintomas de ordem proctológica.

O toque retal na maioria dos casos faz o diagnóstico de compressão extrínsica da parede do canal anal e/ou reto, com mucosa retal sem alterações, em se tratando de lesões císticas.

A RM e TC são indispensáveis na avaliação da lesão, mostrando o tamanho, extensão, invasão de órgãos adjacentes, auxiliando na escolha da abordagem cirúrgica da tumoração.

Em lesões altas e de maior extensão, a via abdominal é preferível, associada ou não a uma abordagem baixa, permitindo melhor visualização de estruturas locais e dissecção mais segura.

A incisão de Parks na abordagem de lesões císticas retro-retais de localização baixa é uma boa opção cirúrgica, com ressecção satisfatória da lesão e sem complicações pós-cirúrgicas nos dois casos aqui relatados.

\section{AGRADECIMENTOS}

Agradecemos o trabalho realizado pela Médica Patologista Dra. Luciana Ximenes, de suma importância para o diagnóstico das lesões aqui apresentadas. 
SUMMARY: Retrorectal tumors are those located in the retrorectal space, derived from germinal layers and may exert extrinsic compression of rectum and anal canal. They are uncommon lesions, usually asymptomatic and more frequent in females than in males. Cystic hamartoma (tailgut cyst) is a congenital retrorectal tumor, derived from embryonic remnants of the posterior gut. The present work makes a review on retrorectal tumors giving emphasis to the cystic lesions and reports two cases of patients suffering from retrorectal cystic hamartoma treated at the Hospital Geral de Goiânia, with evident lesions on rectal examination and imaging showing cystic lesions in the presacral space. The resection of the mass was accomplished by means of a posterior approach (Parks approach) associated with abdominal approach in one of the cases. Mycroscopic section showed cystic hamartoma. Cystic lesions in the retrorectal space usually have a favorable prognosis and the surgical approach will depend on their aspects, main the distance between the lesion itself and the anal verge, and its adherence to surrounding parts in the presacral space.

Key words: Cystic hamartoma, tailgut cyst, retrorectal tumor

\section{REFERÊNCIAS BIBLIOGRÁFICAS}

1. Glasgow SC, Birnbaum EH, Lowney JK, Fleshman JW, Kodner IJ, Mutch DG et al . Retrorectal tumors: a diagnostic and therapeutic challenge. Dis Colon Rectum. 2005; 48:15811587.

2. Dahan H, Arrivé L, Wendum D, le Pointe HD, Tubiana JM. Retrorectal developmental cysts in adults: Clinical and radiologic-histopathologic review, differential diagnosis, and treatment. RadioGraphics. 2001;21:575-584.

3. Wolpert A, Beer-Gabel M, Lifschitz O, Zbar AP. The management of presacral masses in the adult. Tech Coloproctol. 2002; 6(1):43-49.

4. Hannon J, Subramony C, Scott-Conner CE. Benign retrorectal tumors in adults: the choice of operative approach. Am Surg. 1994;60(4):267-72.

5. Lev-Chelouche D, Gutman M, Goldman G, Even-Sapir E, Meller I, Issakov J et al . Presacral tumors: A practical classification and treatment of a unique and heterogenous group of diseases. Surgery. 2003;133(5):473-478.

6. Hjermstad BM,Helwig EB. Tailgut cysts:report of 53 cases. Am J Clin Pathol. 1988; 89(2):139-147.

7. Ito M, Kase H, Shimoyama O, Kobayashi K, Hirano K, Ihara $\mathrm{F}$ et al . Review of three cases with surgical resection of a coccygeal cyst. Journal of the Japan Society of Coloproctology. 2004; 57(1): 28-33

8. Moritani Y. A case of a tailgut cyst. Journal of the Japan Society of Coloproctology. 2002; 55(1): 27-31

9. Prasad AR, Amin MB, Randolph TL, Lee CS, Ma CK. Retrorectal cyst hamartoma: report of 5 cases with malignancy arising in 2. Arch Pathol Lab Med. 2000; 124(5):725729

10. Horenstein M, Erlandson RA, Gonzales-Cueto,DM, Rosai J. Presacral carcinoid tumors : report of three cases and review of literature. Am J Surg Pathol. 1998; 22(2): 251-255

11. Theunissen P, Fickers M, Goei R. Primary large cell neuroendocrine carcinoma of the presacral region. J Clin Pathol. 2001;54(11)880-882.
12. Kim MJ, Kim WH, Kim NK, Yun MJ, Park YN, Lee JT et al . Tailgut cyst : multilocular cyst appearance on MRI. J Comput Assist Tomogr. 1997; 21(5) 731-732

13. Williams LS, Rojiani AM, Quisling RG, Mickle JP. Retrorectal cyst-hamartomas and sacral dysplasia : MR appearance. Am J Neuroradiol. 1998; 19:1043-1045.

14. Menassa-Moussa L, Kanso H, Checrallah A, Abboud J, Ghossain M. CT and MR findings of a retrorectal cystic hamartoma confused with an adnexal mass on ultrasound. Eur Radiol. 2005; 15:263-266.

15. Mourra N, Caplin S, Parc R, Flejou JF. Presacral neuroendocrine carcinoma developed in a tailgut cyst. Dis Colon Rectum. 2003;46(3):411-413.

16. Fujitaka T, Nakayama H, Fukuda S, Ojima Y, Toyota K, Dohi K. A tailgut cyst found accompanying rectal cancer: report of a case. Surg Today (Historical Archive). 1995; 25(1): 65-67.

17. Piura B, Rabinovich A, Sinelnikov I, Delgado B. Tailgut cyst initially misdiagnosed as ovarian tumor. Arch Gynecol Obstet. 2005; 272(4): 301-303.

18. Satyadas T, Davies M, Nasir N, Halligan S, Akle CA. Tailgut cyst associated with a pilonidal sinus: an unusual case and review. Colorect Dis.2002; 4(3):201-204

19. Sobrado CW, Mester M, Simonses OS, Justo CR, Abreu JN, Habr-Gama A. Retrorectal tumors complicating pregnancy. Report of two cases. Dis Colon Rectum. 1996;39:1176-9.

20. Habr-Gama A, Vieira MJF, Marchan LA, Rodriguez JA, Sousa Jr AHS, Jatobá PP et al. Tumores retro-retais no adulto.Descrição de cinco casos. Rev Bras Coloproct. 1986; 6(1):28-36

21. Garcia-Aguilar J. Retrorectal tumors. Am Soc Col Rect Surg. 2001- Care Subjects. Available from: URL: http:// www.fascrs.org/displaycommon. $\mathrm{cfm}$ ? $\mathrm{an}=4$

22. Rosati R, Gravante GP, Pietrasanta D, Cantarelli D, Craboledda P, Filingeri V et al . Brief report: tailgut or teratoma? Progress Reports.2001;1:49-52.

23. Moreira AL, Scholes JV, Boppana S, Melamed J. p53 mutation in adenocarcinoma arising in retrorectal cyst hamartoma(Tailgut Cyst) : report of 2 cases - an immunohis- 
tochemistry / immunoperoxidase study. Arch Pathol Lab Med. 2001; 125(10)1361-1364.

24. Jao SW, Beart RW, Spencer RJ, Reiman HM, Ilstrup DM . Retrorectal tumors: Mayo Clinic experience, 1960-1979. Dis Colon Rectum. 1985; 28:644-652.

25. Bohm B, Milsom JW, Fazio VW, Lavery IC, Church JM, Oakley JR. Our approach to the management of presacral tumors in adults. Int J Colorectal Dis. 1993; 8(3):134-138.

26. Hobson KG, Ghaemmaghami V, Roe JP, Goodnight JE, Khatri VP. Tumors of the retrorectal space. Dis Colon Rectum. 2005; 48:1964-1974.

27. Konemitsu T, Kojima T, Yamamoto S, Koike A, Takeshige K, Naruse T . The trans-sphincteric and trans-sacral approaches for the surgical excision of rectal an presacral lesions. Surg Today.1993;23(10)860-866.
28. Pidala MJ, Eisenstat TE, Rubin RJ, Salvati EP. Presacral cysts: transrectal excision in select patients. Am Surg. 1999;65(2)112-115.

\section{Endereço para correspondência:}

GABRIELLA O. FERNANDES

Hospital Geral de Goiânia - H.G.G.

Serviço de Coloproctologia

Av: Anhanguera, No. 6379 - Setor Oeste

74043-011 Goiânia - GO

E-mail: gabicir@ig.com.br 